\title{
Significance of the Sampling Errors due to Changing Land-sea Distribution in the Phanerozoic Paleo-climate Reconstruction
}

\author{
Naomi SAKAMOTO \\ Graduate School of Science and Technology, Kobe University, Kobe, Japan \\ and \\ Kooiti MASUDA \\ Frontier Research System for Global Change, Yokohama, Japan \\ (Manuscript received 18 January 2000, in revised form 30 January 2002)
}

\begin{abstract}
Fossil climate indicators are often quite fragmental. Further, sampling points of fossil indicators do not have uniform distribution. Latitudinal distribution of the terrestrial area are changing because of plate tectonics. All these circumstances may bring about strongly biased set of sampling points, which in turn cause errors in the reconstruction of the paleo-climate during the Phanerozoic.

If the number of the sample points equals 100 or less, and when there is no information on altitude of each sample point, our simulations estimate that inferred global average surface temperature is accompanied with an uncertainty of more than $2 \mathrm{~K}$. Results of our simulation indicates the inferred temperature can strongly vary with the change of the land-sea distribution.

This result suggests that the reconstructed climate change in precedent studies can be a result of the varying land-sea distribution under the same global climatic condition during the Phanerozoic.
\end{abstract}

\section{Introduction}

Reconstruction of climatic variation during the Earth's history has often been based on the geographical distribution of evidence of ice or other fossil climate indicators (e.g., Frakes et al. 1992; Crowley and North 1991). In addition, temperature changes during the Phanerozoic (the last 600 million years) have often been reconstructed from the concentration of isotopes (e.g., Dickens et al. 1995; van Eijden and Ganssen 1995).

Corresponding author: Naomi Sakamoto, Graduate School of Science and Technology, Kobe University, Nada-ku, Kobe 657-8501, Japan.

E-mail: sakamoto@shizen.sci.kobe-u.ac.jp

(C) 2002, Meteorological Society of Japan
However, these kinds of reconstruction of climatic variation does not always guarantee a good representation of the surface temperature of the Earth, because these fossils or isotopes are indicators of temperature only where and when the materials were supplied to sediments. Thus, without the evaluation of the location of samples, we can hardly reconstruct the history of climate during the Phanerozoic (Sakamoto and Masuda 1999). For example, we cannot always differentiate glacial deposits in high altitude under global warm climate, and those in low altitude under global cool climate (Sakamoto et al. 1998).

It is a well known fact that the land-sea distribution has been changing through the geological time scale (e.g., Scotese and McKerrow 1990). Fossils indicating the past climate have often been destroyed by tectonic or other activ- 
ities, and therefore, our information source of climate is mainly restricted on the portion of the plate that has not been destroyed by subduction process. Strata containing fossils are not always exposed, reducing the chance for finding them. Thus, there are few number of fossils available for the reconstruction of climates.

The geographic distribution of fossils is influenced by the changing land-sea configuration. In particular, all the fossil information (both of past land and past ocean) older than 200 million years, are available only on the present continental crust, because the oceanic crust of those ages have been lost by subduction. Also, evidence of permanent ice, one of the most often used types of climate indicators (e.g., Hambrey and Harland 1981), is available only over past land.

Thus, we should operate with modest quantity of fossils, distributed inhomogeneously, to infer the paleo-climate, which fact may bring large uncertainty in the inference of paleoclimate. In this paper, we estimate the significance of the sampling error caused by changing land-sea distribution during the Phanerozoic paleo-climate reconstruction.

\section{Observation system simulation experiments (OSSE) for the paleo-climate}

\subsection{Method and data}

In this paper, we use the term "fossil climateindicator" or simply "fossil" to mean the geological evidence of the past climate, of organic as well as inorganic nature.

We made a series of statistical calculation to estimate effects of a) paucity of the information; b) uncertainty of altitude of the provenance of the fossil as well as; c) changing land-sea distribution pattern on the inference of paleoclimate. We assume a certain hypothetical state of climate as 'true', and try to reconstruct it with a limited number of samples. This kind of methodology is often employed in meteorological contexts (Oort 1978; McConnel and North 1987) and called 'observation system simulation experiments'.

First, for our estimation, we prepared a data set of annual mean surface temperature with resolution of $0.5^{\circ}$ latitude/longitude, representative of the present climate. The values contained in the data set are not actually observed values at each grid points, but values interpolated from the existing network of observing stations. However, for the purpose of our experiments, we assume that those are the 'true' annual mean temperature at each of the grid points.

Grid points on the continents were filled with the values of Leemans and Cramer (1991). They first reduced temperature observed at stations to the sea level, assuming a constant lapse rate, next horizontally interpolated them to grid points, and then converted them back to the land elevation of the grid points assuming the same lapse rate. They did not, however, give values over oceans or over Antarctica. Oceanic and Antarctic grid points were filled with the values of Legates and Willmott (1990). They made two-dimensional interpolation from stations to grid points without explicitly taken land elevation into account. We consider the former relatively suitable for our purpose, even though their crude assumption of constant lapse rate. On the other hand, we consider that the temperature field of the latter over Antarctica is, though distorted geographically, also good enough for our purpose, because it reflects observations at stations on the ice sheet and topography is relatively smooth there.

We picked up samples from this known dataset in order to simulate the process to reconstruct paleo-climate. Comparing statistics obtained from limited samples against the knowledge of the 'true' field in our experiments, we estimated how the inference on paleoclimate will be distorted by: 1) small number of samples; 2) uncertainty in altitude; and, 3) pattern of land distribution when the sampling is limited over land.

We picked up certain numbers of points, at random, from the above data set. We calculated global mean temperature, and inferred the location of ice margin from the data points picked up. In this context, 'ice margin' means the margin of latitudinal distribution of occurrence of permanent ice. Finally, we compared results of our experimental calculations with the above defined "true values".

\subsection{Reconstruction of present condition of the climate by OSSE}

In this section, we present the sensitivity of this method. 


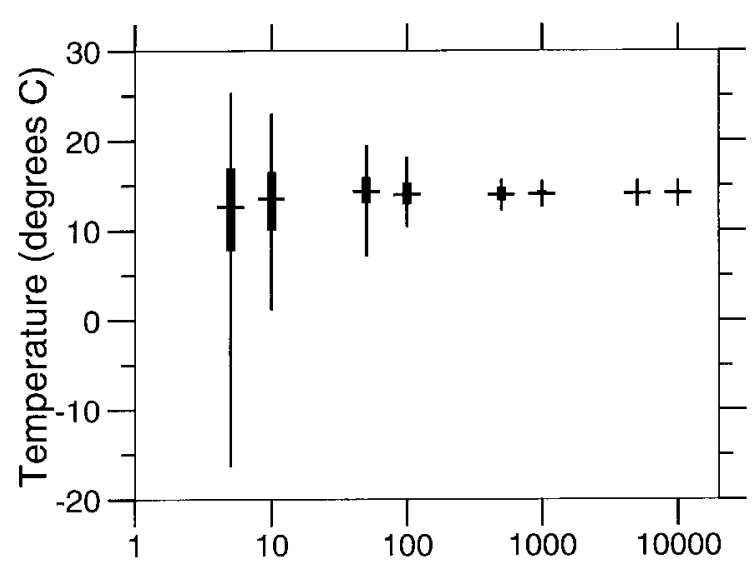

Fig. 1. Mean (horizontal bar), 25\% and $75 \%$ rank values of the samples (thick vertical bar), minimum and maximum (thin vertical bar) of $m(=100)$ estimate of "global mean temperature" for each $n(=5,10,50,100,500,1000,5000$, 10000). $\mathrm{X}$-axis is $n$ and $\mathrm{Y}$-axis is global mean temperature $\left({ }^{\circ} \mathrm{C}\right)$.

\section{a. Effect of the number of the samples}

From the data set described previously, we picked up $n$ points $(n=5,10,50,100,500,1000$, $5000,10000)$ at random with probability proportional to representative area of grid boxes. Such sampling was repeated $m(=100)$ times for each $n$. We regarded the average of temperature for $n$ points as the inferred global mean temperature. Figure 1 shows that the effects of the number of the samples on the reconstruction of global mean temperature. Mean, 25\% and $75 \%$ rank (quartile) values of the sample, minima and maxima of $m$ estimates of "global mean temperature" for each $n$ are shown in this figure. Our results of the calculation do not always have normal distribution, we use $25 \%$ and $75 \%$ rank values of $m$ instead of the standard deviation as an indicator of the distribution of our results. When $n=100$, temperature range between $25 \%$ and $75 \%$ rank values is about $2.5 \mathrm{~K}$. In this experiment, sampling points on marine as well as continental area have been incorporated, because the aim of the experiment is to show the effect of the paucity of sample number. The effect of the bias towards land area in the distribution of fossil records is discussed separately in Subsection 2.3.

Figure 1 shows that sampling error of the temperature estimated by 100 or less was larger than $2.5 \mathrm{~K}$. Thus, if there is any single evidence that indicates cooler condition not much exceeding this magnitude, this evidence should be accepted as indicating 'local' cool condition, not always cooler condition on the global scale.

Generally, in paleo-climatic reconstruction studies, the amount of data points referred for each time slice is about 100 or less (Frakes et al. 1992). Therefore, we carried out our experiments with 100 points. The effect of the number of samples to the uncertainty of paleo-climatic inference should be taken into account in the following experiments as well, though not explicitly calculated there.

\section{b. Effects of the elevation}

Variation of the altitude on land at the present is between $-400 \mathrm{~m}$ (coast of the Dead Sea) and $8848 \mathrm{~m}$ (top of Qomolangma). Temperature strongly varies with the altitude. Though it is not actually constant, it may be approximated by the typical value of temperature decrease in the troposphere, $0.65^{\circ} \mathrm{C}$ for every $100 \mathrm{~m}$ height increase (U.S. Standard Atmosphere, NOAA et al. 1976).

Over the geological time scale, we have little information on paleo-altitude. Thus, the effects of the paleo-altitude has been seldom discussed in the studies of the reconstruction of the past climate. We calculated the effect of uncertainty of the altitude in reconstruction of paleoclimate in which the topography is totally different from present and is not known for certain.

To estimate the altitude effect, we at first prepared a hypothetical global sea-level air temperature distribution from the data set used for the first experiment. For this purpose we calculated a distribution of temperature reduced to the sea level (Fig. 2). In this and following sections, we use this data-set for our calculation. The sea-level temperature value at each latitude is calculated as the average of the temperature values at all the data points reduced to the sea surface at the latitude irrespective of hemispheres. As a result we prepared a virtual globe with 360 temperature zones with the width of $0.5^{\circ}$ latitude running parallel, each zone of which having one specific temperature value. To estimate the effect of 


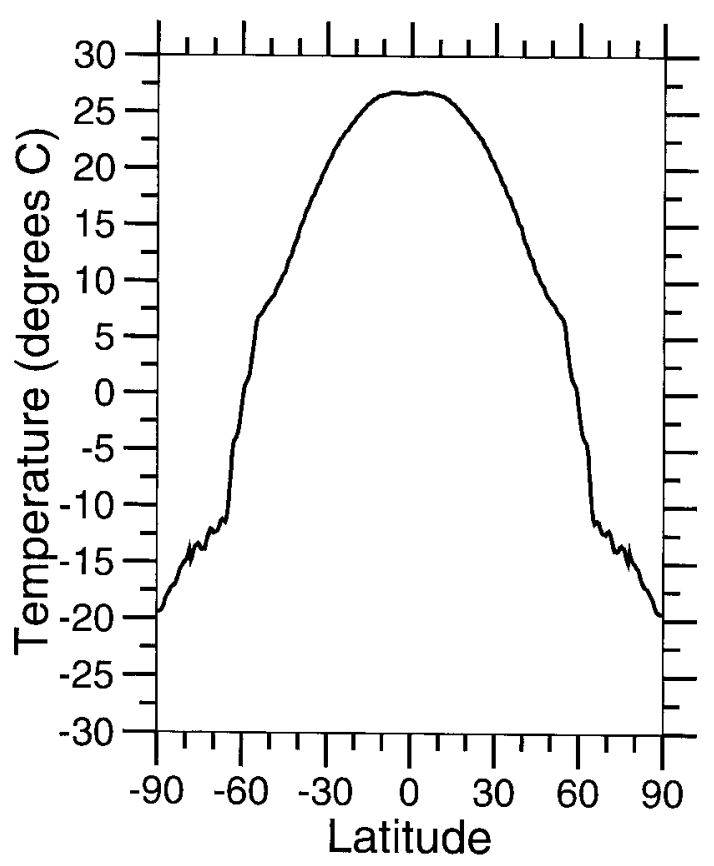

Fig. 2. Latitudinal distribution of the temperature used in this calculation. To get normalized data value to the sea surface, we computed averages of temperatures at grid points on the sea level at every one half degree of latitude irrespective of hemispheres. We consider this dataset as "temperature at the sea level".

altitude, we calculated two values for each of $m=100$ runs of experiments.

At first we picked up $n=100$ sample points from the above prepared data set. We call this data set "temperature at sea level". We calculated an average value of temperature for these samples. This average sea level temperature could be regarded as global mean temperature neglecting the effect of the altitude.

We then gave altitude for each of the $n=100$ samples at random with the frequency distribution of the present topography. Then for each of the $n=100$ samples, temperature correction was carried out as follows.

$$
\begin{aligned}
T & =T_{0}-\Gamma H, \\
\Gamma & =6.5 \mathrm{~K} / \mathrm{km},
\end{aligned}
$$

where $T, T_{0}$ and $H$ are temperature including the effect of the altitude, temperature at the sea level picked up from the dataset, and altitude, respectively.

Finally we calculated the average of $n=100$ corrected values. This corrected value could be considered as the mean global temperature including the synthesized altitude effect.

We carried out the above procedure $m=100$ times.

The inferred global mean temperature at sea level is $15.9^{\circ} \mathrm{C}$, and that including the altitude effect is $14.0^{\circ} \mathrm{C}$. The global mean temperature neglecting the effect of the altitude is $1.9 \mathrm{~K}$ higher than the global mean temperature including the altitude effect.

In the context of reconstruction of the past climate, our result suggest the following issues that seem to have seldom been taken into account. If we take the global mean surface temperature as the target variable of reconstruction, the reconstruction can be biased ( positively or negatively) due to difference between frequency distributions of paleo-altitude of the fossil climate indicators and that of the topography. If we take global mean sea-level temperature as the target variable, we have another source of bias in addition to the above. When the paleo-altitudes of fossil climate indicators are unknown, it is sometimes implicitly assumed that they are near the sea level. This assumption results in a negative bias in the reconstruction of the global mean sea-level temperature. If we tentatively assume that the frequency distribution of altitude of climate indicators in the past is the same as that of the present topography, this bias is about $-2 \mathrm{~K}$.

\subsection{Estimation of sampling errors during the Phanerozoic}

In this section, we estimate significance of the sampling errors. For ease of calculation, we neglected the effect of seasonal cycle.

\section{a. Effects of the changing continental distribution}

As discussed earlier, much of information on paleo-climate during the Phanerozoic is better conserved on land area. Thus, the changing land-area distribution through the geological time may bring about biases in reconstructing the paleo-climate. We estimated the effects of the land-sea distribution on the paleo-climate reconstruction during the Phanerozoic. To illustrate the effect of land-sea distribution 


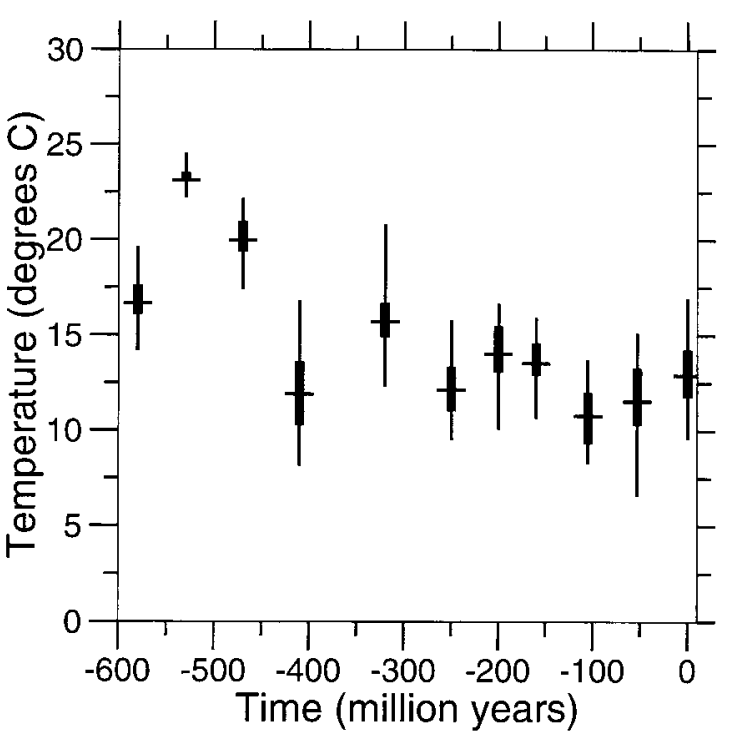

Fig. 3. Mean (horizontal bar), 25\% and $75 \%$ rank values of samples (thick vertical bar), minimum and maximum (thin vertical bar) of estimate of "mean temperature on the land" according to the changing land-sea distribution. Xaxis is time (million years) and Y-axis is global mean temperature $\left({ }^{\circ} \mathrm{C}\right)$.

changes on sampling temperature, we picked up $n=100$ points on land at random and estimated global mean temperature for each geological period. We referred to the following paleo-geographical maps for the Phanerozoic land-sea distribution: Scotese and McKerrow (1990) for Paleozoic part, and of Smith et al. (1994) for Mesozoic and Cenozoic part. Random sampling was repeated $m=100$ times for each period.

Figure 3 shows mean, 25\% and $75 \%$ rank, minimum and maximum estimates of the mean temperature calculated from our experiments for the respective geological periods. During the Early Paleozoic (about 600 to 450 million years before present), the calculated temperature is more than $10 \mathrm{~K}$ higher than that of the other periods. During the Early Paleozoic, there was a large land area around the equator. This result indicates that if there is much of the land area around the equator and if there is little land on the polar region, temperature reconstructed in referring to the fossil record from land area is as much as $12 \mathrm{~K}$ higher.

\section{b. Estimation of the latitudinal distribution of the permanent ice}

In the previous paragraphs, we discussed about the sampling influence on the global average temperature. Latitudinal distribution of permanent continental ice is one of the major methods of the reconstruction of the paleoclimate. For the comparison with the precedent reconstructions of the paleo-climate, we examined the effect of changing land-sea distribution on the estimation of latitudinal distribution of the permanent ice.

We assume that there was permanent ice if the temperature was lower than $0^{\circ} \mathrm{C}$ at the sampling point. We also assume that permanent ice was only formed on continents. Therefore, our inferred 'ice margin' is defined as the lowest absolute value of latitude of sampling points with temperature lower than $0^{\circ} \mathrm{C}$ within the set of $n=100$ samples.

We used the dataset of "the temperature at sea-level" consistently, because we do not have enough knowledge of the frequency distribution of land altitude in the past. Random sampling was repeated $m=100$ times at each period.

Figure 4 shows the latitudinal distribution of the ice margin during the Phanerozoic. Figure 4-a is a our estimation by OSSE; Fig. 4-a shows the mean, $25 \%$ and $75 \%$ rank, minimum and maximum of the ice margin during the Phanerozoic. Figure 4-b shows latitudinal ranges of occurrence of evidence of ice (glacial and icerafted deposits) during the Phanerozoic (Frakes and Francis 1988; Most of their data came from Hambrey and Harland 1981).

According to this analysis, more than $75 \%$ data of the Late Cambrian indicates there was no permanent ice. During Carboniferous, both Fig. 4-a and Fig. 4-b shows permanent ice appeared most extensively.

This experiment demonstrated that under the single pattern of the temperature, changing land-sea distribution yields the following apparent tendency;

(a) There was no or little ice during the Late Cambrian. More than $75 \%$ data indicates there was no ice during the Late Cambrian.

(b) Permanent ice extended to the lower latitude from the Early Paleozoic (CambrianOrdovician) to the Latest Paleozoic (Permian).

(c) Ice margin of the Early Mesozoic (Triassic) was located at about 65 degrees of latitude. 
(a)

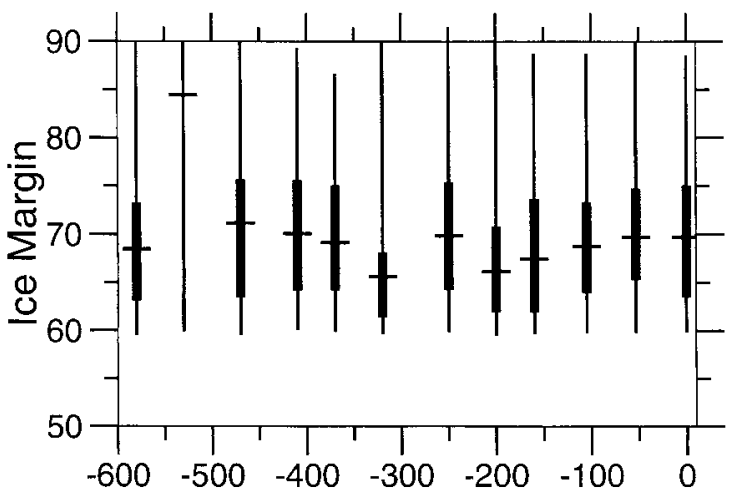

(b)

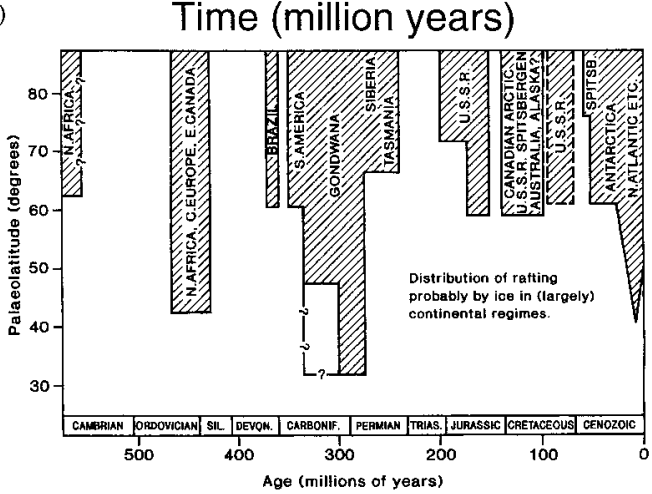

Fig. 4. Latitudinal distribution of the ice margin during the Phanerozoic. $\mathrm{X}$-axis is time (million years) and $\mathrm{Y}$-axis is ice margin (degrees of latitude). Figure 4-a (above) shows mean (horizontal bar), $25 \%$ and $75 \%$ rank values of the samples (thick vertical bar), minimum and maximum (thin vertical bar) of our estimate of the "ice margin" of the different land-sea distribution estimated by OSSE. Figure 4-b (bottom) shows occurrence of paleo-latitude of glacial and ice-rafted deposits (Frakes and Francis 1988).

(d) Ice margin retreated to 70 degrees of latitude from the Early Mesozoic to the Late Mesozoic (Cretaceous).

This apparent tendency (Fig. 4-a) were produced from the same distribution of temperature (Fig. 2) but with different sampling due to varying land-sea distribution. Nevertheless, it fairly well corresponds with the migration of conventionally inferred ice margin of the geological past (Frakes et al. 1992; Huggett 1991).

Changing land-sea distribution, especially of the proportion of the land in the polar region, plays an important role in the reconstruction of temperature and ice margin during the Phanerozoic. We use the same and single dataset of the global temperature distribution for the calculation of the Fig. 3 and Fig. 4-a for all of the respective time slices. It means that 'true' global mean temperature is assumed to be constant in these calculations. Figure 3 and Fig. 4-a indicate that changing land-sea distribution brings about the temperature variation. This sampling error corresponds to uncertainty of about $12 \mathrm{~K}$ of the global mean temperature and 20 degrees of latitude of the ice margin.

\section{Summary}

Sakamoto and Masuda (1999) discussed that temperature reconstructed from a few samples of the fossil or sediments did not necessarily indicate a global representative of the surface temperature. In this paper, we use "global mean temperature" as the most important variable to characterize the climates of a specific geological past, because this parameter is quantitative and can offer the same approach in our calculation though the Phanerozoic.

According to precedent studies of the paleoclimate (Frakes et al. 1992; Huggett 1991) dealing with the latitudinal distribution of the ice margin during the Phanerozoic, global climate was warmer from the Early Paleozoic and cooler during the Late Paleozoic to the Early Mesozoic. Our calculations using the same and the single latitudinal distribution of the temperature under different land-sea distribution patterns show the same trend in the history of the observed ice margin. In our calculation, the global mean temperature during the Phanerozoic was constant. Thus, the temperature changes in our results indicate sampling error caused by the changing land-sea distribution.

These results present the possibility that the reconstructed temporal pattern of the climatic change in precedent studies can be a result of the varying land-sea distribution under the same global climatic condition of the geological past.

As we have shown, the error bars in inferring paleo-climate can be so large, that it will lead to misleading conclusions, the more so when the data are not accompanied with information on paleo-altitude. 
Further, even under a single global climatic regime, if we are compelled only to refer to landbased data, a clear phantom paleo-climatic fluctuation may emerge, as shown in our analysis incorporating various distributional patterns of drifting continents. An overall similarity of this phantom climatic fluctuation with those obtained by Frakes et al. (1992) and Huggett (1991) raises a grave alarm to the conventional procedure of reconstructing paleo-climatic data.

We hope that in the future paleo-climatic studies, following warnings resulting from our demonstrations should be seriously taken into consideration to minimize error-bars of paleoclimate reconstructions:

1) Number of samples should be large enough to yield a statistically significant result;

2) We need to check out if data set with heterogeneous altitude are not mixed together; and,

3) It is necessary to sample paleo-data uniformly with respect to paleo-latitude to avoid too many or too few samples representing a certain latitudinal zone.

\section{Acknowledgments}

We thank Prof. Taroh Matsuno (Frontier Research System for Global Change) and Prof. Terufumi Ohno (The Kyoto University $\mathrm{Mu}-$ seum) for reading our manuscript.

Gridded climatic data are taken from the CD-ROM 'Global Ecosystems Database, Disc A' published by the U.S. National Geophysical Data Center in 1992.

\section{References}

Crowley, T.J. and G.R. North, 1991: Paleoclimatology. Oxford University Press.

Dickens, G.R., J.R. Neil, D.K. Rea and R.M. Qwen, 1995: Dissociation of ocenanic methane hydrate as a cause of the carbon isotope excursion at the end of the Paleocene. Paleoceanography, 10, 965-971.

Frakes, L.A. and J.E. Francis, 1988: A guide to Phanerozoic cold polar climate from high-latitude ice-rafting in the Cretaceous. Nature, 333, $547-549$.
- $\longrightarrow$ and J.I. Syktus, 1992: Climate Modes of Phanerozoic. Cambrige University Press.

Hambrey, H.A. and W.B. Harland, eds., 1981: Earth's Pre-Pleistocene Glacial Record. Cambridge University Press.

Huggett, R.J., 1991: Climate, Earth Processes and Earth History. Springer-Verlag.

Leemans, R. and W.P. Cramer, 1991: The IIASA database for mean monthly values of temperature, precipitation and cloudiness of global terrestrial grid. Research Report RR-91-18, International Institute of Applied Systems Analyses, Laxenburg. 61 pp.

Legates, D.R. and C.J. Willmott, 1990: Mean seasonal and spatial variability in global surface air temperature. Theoretical and Applied Climatology, 41, 11-21.

McConnell, A. and G.R. North, 1987: Sampling errors in satellite estimates of tropical rain. $J$. Geophys. Res., 92, 9567-9570.

NOAA, NASA and U.S Air Force, 1976: U.S. Standard Atmosphere 1976, U.S. Government Publishing Office.

Oort, A.H., 1978: Adequacy of the rawinsonde network for global circulation studies tested through numerical model output. Mon. Wea. Rev., 106, 174-195.

Sakamoto, N. and K. Masuda, 1999: Climatic fossilMaking of the uniform dataset of the numerical simulation and observation in the paleoclimatology. Kagaku, 69, 790-793 (in Japanese).

Sakamoto, N., C. Suzuki and K. Masuda, 1998: An attempt toward the reconstruction of paleoclimate by means of a data base of fossils. Geoinformatics, 9, 13-18 (in Japanese).

Scotese, C.R. and W.S. McKerrow, 1990: Revised world map and introduction, Paleozoic Paleogeography and Biogeography (Eds. W.S. McKerrow and C.R. Scotese), Geological Society Memoir, 12, 1-21.

Smith, A.G., D.G. Smith and B.N. Funnell, 1994: Atlas of Mesozoic and Cenozoic Coastlines. Cambridge University Press.

van Eijden, A.J.M. and G.M. Ganssen, 1995: An Oligocene multi-species foraminiferal oxygen and carbon isotope record from ODP Hole 758A (Indian Ocean): paleocenographic and paleo-ecologic implications. Marine Micropaleontology, 25, 47-65. 